\title{
On the parameterization of single and binary stars
}

\author{
O. Malkov, S. Sichevskij, D. Kovaleva and V. Myakutin \\ Institute of Astronomy, 48 Pyatnitskaya St., 119017, Moscow, Russia \\ email: malkov@inasan.ru
}

\begin{abstract}
Many astrophysical problems in the theory of formation and evolution of stars and stellar groups require knowledge of the mass or age of individual stars. Stellar masses and ages are not directly observable parameters, and most of the methods used to determine them are based on the calibrating relations obtained from observations or from theoretical considerations. In most cases, the mass-luminosity relation, based on the masses of approximately two hundred well-studied binaries, is the only way to estimate the mass of single stars. Unfortunately, the statistical nature of the mass-luminosity relation means that the stellar masses determined with it may only be considered as approximate. The establishment of methods for estimating stellar masses and ages of stars with accuracy comparable to direct methods remains a challenging task. This work proposes a way to use the location of a star in the HR diagram to estimate its mass and age based on modern stellar models. The method is based on the geometric similarity of evolutionary tracks for the stars at the same evolutionary stage. To examine the proposed approach and involved assumptions, it has been applied to various test data sets.
\end{abstract}

Keywords. stars: fundamental parameters, stars: binaries, stars: evolution

\section{Method of determination of the mass and the age of the star}

According to the evolutionary calculations, for the stars of equal evolutionary status and different masses

$$
\log T_{\text {eff }} \propto \log m, \quad \log L \propto \log m, \quad \log t \propto \log m,
$$

where $T_{\text {eff }}$ is the effective temperature of the star, $t$ is its age, $L$ and $m$ are respectively luminosity and mass in solar unit. The relations (1.1) allow determination of the mass and age for any point at the line of equal evolutionary status.

However, the published calculations of stellar evolution usually prevent direct application of equations (1.1), as the grid of evolutionary models in the HR diagram has irregular structure and needs recalculation to cover the diagram evenly. Another thing is, the relations (1.1) are valid for the stars of equal evolutionary status. This is the main reason that restructuring the grid of evolutionary tracks is necessary. There are various approaches to determine the evolutionary status of a star. One of them (Myakutin \& Piskunov 1995) is as follows. One needs to select the same evolutionary stages (for example, the stage of hydrogen burning in the core) in the tracks of stars of various masses. The resulting segments are congruent in the HR diagram. Tracks at this stage are divided into equal number of intervals. In this scheme, evolutionary interval limits make a sufficiently good approximation to the lines of equivalent evolutionary status. To make the estimation of stellar masses and ages much more effective, one can unite several stages characterized by the same process of energy release which results in the so called evolutionary phase of the HR diagram. The phase is characterized by the energy source, typical mass range and region of the HR diagram. All the tracks of the same evolutionary phase have the same structure, and are congruent. 


\section{Application of the method to single and binary stars}

A set of selected BaSeL (Lejeune et al. 1997) data has been used to test the method. The short statistics of their processing is as follows: of the total 467 of synthetic spectra, $228(49 \%)$ allowed to estimate mass and age of a star; 50 of them resulted in multiple solutions.

The calculated stellar characteristics were used to construct mass - age, mass radius, mass - effective temperature relations. The analysis of these relations allows us to conclude that the method leads to predictable results, and the uncertainties of the calculated characteristics are distributed rather smoothly. The regions of the HR diagram where evolutionary tracks are thickening naturally lead to less accurate estimates of stellar parameters.

The main conclusion of the tests of this method with the BaSeL library data is that the method allows uniform estimates of stellar masses, ages and radii with a predictable uncertainties distribution.

A well known fact is that the eclipsing binaries showing lines of both components in the spectrum (double-lined eclipsing binaries) allow rather accurate estimates of their physical characteristics. Thus, these stars provide a valuable tool for examination of the described method. We have compared the observational masses of the binary components with the calculated ones.

The uncertainties of the stellar masses estimated with the proposed method are comparable with the accuracy of ones obtained from direct observations. This allows us to recommend the method for estimates of masses of single stars by the localization in the HR diagram. We note, however, some systematic trend toward overestimation of masses obtained with theoretical models for low mass stars, respectively to observed ones. This corresponds to the systematic shift of the tracks in comparison with observations (overestimated radii, underestimated effective temperatures) noted recently by several authors.

As for the ages, the estimates for intermediate mass stars are more reliable, while those obtained for low mass stars are very uncertain, due both to slower movement of these stars in the HR diagram with age at stages close to the main sequence, and to certain disagreements between theoretical models for this mass range.

The proposed method can be applied to data to be generated from future ground-based and space observatories (Gaia).

Acknowledgments. We are greatful to Coryn Bailer-Jones for his support during the method testing. This work has been supported by Russian Foundation for Fundamental Research grants 08-02-00371 and 09-02-00520, by the Federal Science and Innovations Agency under contract 02.740.11.0247 and by the Presidium RAS program "Leading Scientific Schools Support" 4354.2008.2. This research has made use of the SIMBAD database, operated at the Centre de Donnees astronomiques de Strasbourg, and of NASA's Astrophysics Data System Bibliographic Services.

\section{References}

Lejeune, Th., Cuisinier, F., \& Buser, R. 1997, A\&AS, 125, 229

Myakutin, V. I. \& Piskunov, A. E. 1995, Astronomy Reports, 39, 316 